\title{
On the relations between implicit and explicit spatial binding: Evidence from Balint's syndrome
}

\author{
CATERINA CINEL \\ University of Essex, Colchester, England \\ and \\ GLYN W. HUMPHREYS \\ University of Birmingham, Birmingham, England
}

\begin{abstract}
We report two experiments examining whether factors that affect binding in explicit report tasks also affect implicit binding in a patient with Balint's syndrome, G.K. Replicating prior studies, we showed that there could be implicit binding of visual features in a patient with Balint's syndrome who was at chance at explicitly discriminating the relations between the features. Nevertheless, when color-form relationships were coded, we showed that G.K.'s implicit binding, as well as his explicit report of binding relationships, was affected by the spatial distance between the stimuli. Similarly, we demonstrated effects of grouping on both implicit and explicit binding of the spatial relations between shapes. We interpret the qualitative similarities between implicit and explicit binding as indicating that they reflect a single process in which binding relations, initially established preattentively, are later consolidated by attention. This later process, of attentional consolidation, is disrupted in Balint's syndrome.
\end{abstract}

There is good evidence that many basic properties of visual stimuli-their color, direction of motion, and shape - are processed independently and in parallel (see Heywood \& Cowey, 1999, and Heywood \& Zihl, 1999, for neuropsychological evidence). Hence, to see a coherent world, in which color, motion, and shape are linked correctly, the brain may need to bind together the independently processed features (see Robertson, 2003, for a recent review). There are several accounts of how this binding process may take place. For example, binding may depend on temporal synchrony of firing between neurons responding to the properties of a common object (e.g., Singer \& Gray, 1995). Alternatively, it may depend on some common registration of features within some master map of locations and/or on the application of serial attention to that map (as in Treisman's feature integration theory; Treisman, 1998).

Neuropsychological evidence has linked the binding process to regions of the parietal lobe (e.g., Friedman-Hill, Robertson, \& Treisman, 1995) and the pulvinar (Ward, Danziger, Owen, \& Rafal, 2002). For example, following bilateral parietal damage, there can be marked problems with feature binding, so that patients frequently misattribute the features of one object to another (e.g., reporting a

This work was supported by grants from the MRC and the Stroke Association to the second author. We thank G.K. for his continuing kind participation and Patricia Reuter-Lorenz, Asher Cohen, Eva Wojciulik, and an anonymous referee for comments on a previous version of the manuscript. Correspondence concerning this article should be addressed to G. W. Humphreys, Behavioural Brain Sciences Centre, School of Psychology, University of Birmingham, Birmingham B15 2TT, England (e-mail: g.w.humphreys@bham.ac.uk). red $\mathrm{X}$ and blue $\mathrm{O}$ as a red $\mathrm{O}$ and a blue $\mathrm{X}$; Friedman-Hill et al., 1995; Humphreys, Cinel, Wolfe, Olson, \& Klempen, 2000). These illusory conjunctions (ICs) can also be found in patients with unilateral damage when the stimuli are presented within the contralesional field (A. Cohen \& Rafal, 1991). The ICs may reflect the coarse spatial coding available to these patients, so that (say) color information may overlap the spatial location recorded for a different shape, leading to an incorrect binding of color and shape (Humphreys et al., 2000). It may also reflect the difficulty such patients have in attending to spatial location. Attention may normally serve to filter out irrelevant stimuli (cf. Moran \& Desimone, 1985). For patients with difficulties in visual attention, the irrelevant information may remain available to be bound incorrectly across stimuli.

Despite the fact that patients with parietal damage can make binding errors (ICs) when asked to explicitly report stimuli, there is also evidence that the same attributes can be bound implicitly, when the patients do not have to report the relations between the attributes. Evidence on implicit binding in a Stroop task was reported by Wojciulik and Kanwisher (1998) for R.M., a patient with Balint's syndrome following bilateral parietal damage. FriedmanHill et al. (1995) found that R.M. made abnormal numbers of ICs between colors and shapes when asked to identify multiple colored letters. Despite this, Wojciulik and Kanwisher demonstrated that R.M. was sensitive to Stroop interference, suggesting that color and form information was bound together, at least at some stage. Their procedure is highly relevant to the present study, and so we will describe it in some detail. In their variant of the Stroop task, Wojciulik and Kanwisher presented two words, one above the other, on a black background. One word was colored, 
and the other was white (see Figure 1A). The task was to name the nonwhite color as quickly as possible. There were two critical conditions. In the same object inconsistent (SOI) condition, the color name word and its color were inconsistent (e.g., the color name was PURPLE and its color brown); the white word was consistent with the color (e.g., the word was BROWN). In the different object inconsistent (DOI) condition, the white word was inconsistent with the color, and the colored word was consistent (e.g., the white word was PURPLE; the colored word had a matching color and name- - e.g., BROWN name and brown color). R.M. was slower to name the color in the SOI condition than in the DOI condition. In other blocks of trials run within the same study, R.M. was asked to read the colored word (words with color-neutral meanings were then used, to reduce confusions). This last task required R.M. to have explicit knowledge of which word was bound with which color. In this explicit binding task, R.M. performed at chance; he was as likely to read the word in white as the (nonwhite) colored word. Similar results with R.M. have been reported by Robertson, Treisman, FriedmanHill, and Grabowecky (1997), using a spatial variant of the Stroop task. This time R.M. was presented with the

\section{A) IMPLICIT BINDING TASK}
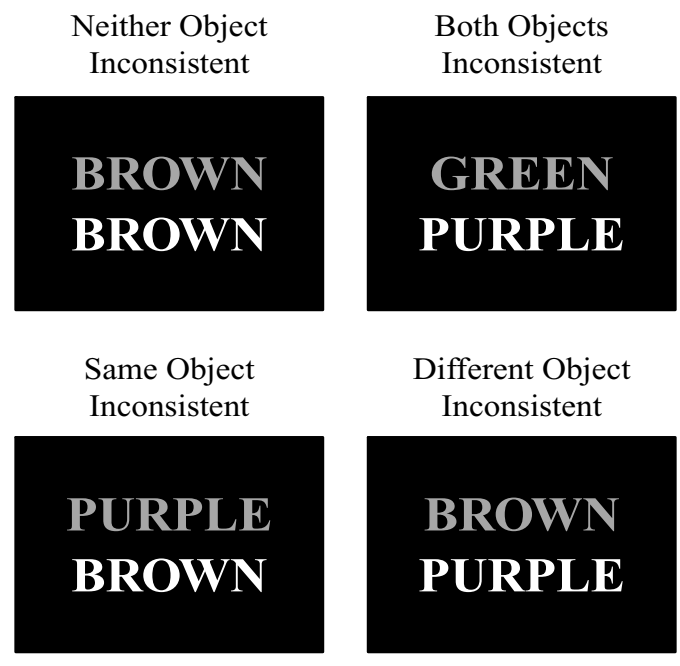

Different Object Inconsistent

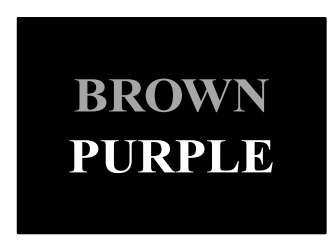

B) EXPLICIT BINDING TASK
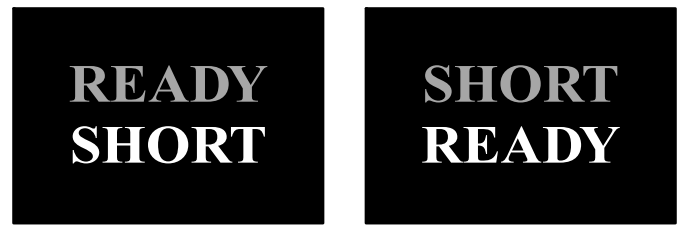

Figure 1. Examples of the displays in Experiment 1A. (A) Conditions used in the implicit binding task (the task was to name the color). (B) Conditions used in the explicit binding task (the task was to read the colored word). Shading indicates that the word, in this particular example, was shown in the color brown. words UP or DOWN located at the center, at the top, or at the bottom of a rectangular frame. In an explicit localization task, in which strings of Xs were presented, R.M. performed at chance (are the strings of letters at the top, center, or bottom of the box?). However, when the words UP or DOWN were presented and the task was to read the word as rapidly as possible, R.M. showed spatial Stroop interference: He was slower to read the word when it was presented in the location inconsistent with the meaning of the word (e.g., the word UP presented at the bottom of the rectangle) than when the word was consistent with its spatial location (UP at the top of the rectangle).

Other data on implicit binding in a patient with Balint's syndrome have been documented by Humphreys, Riddoch, Nys, and Heinke (2002). Their study was conducted with G.K., the patient who was also the subject of the present study. They briefly presented one or two letters to G.K., who was asked to report the letter identities. Humphreys et al. (2002) reported that G.K. was poor at identifying a single letter presented to his left visual field (relative to letters presented to his right field). Interestingly, his report of left-side letters improved when the left letter appeared simultaneously with a letter in the right field. This was interpreted as being due to temporal binding based on synchrony of onset between the two letters (cf. Singer \& Gray, 1995). Report of the left letter was improved if it was bound to the right letter, since attention was then attracted to the pair of letters as a perceptual group. When presentation of the letters was staggered, so that they did not onset together, report of the left letter decreased even on two-item trials. Despite this improved report under simultaneous presentation conditions, G.K. consistently judged that the right item preceded the left when they onset at the same time. This prior entry effect was overcome only when the left letter preceded the right by $750 \mathrm{msec}$. Thus, there was a dissociation between G.K.'s explicit knowledge of simultaneous onsets and his ability to use the onsets for temporal binding. Humphreys et al. (2002) also found that as letter presentation times increased, there was a decrease in the benefit for two-item trials over trials on which only the left letter appeared. They attributed this to the binding signal's decaying over time, unless reinforced by the letters having a common offset (under short-presentation conditions). As a consequence, G.K. was no longer able to attend to items as a single group.

Although several studies have now demonstrated implicit binding in patients with parietal damage and poor explicit binding, we as yet know little about the relations between these different binding processes. Is implicit binding qualitatively different from explicit binding, or is there a single process that is affected differentially by the requirement to explicitly report the properties present (e.g., the recruitment of spatial attention)? In the present study, we attempted to examine implicit binding in Balint's syndrome in more detail. We investigated whether implicit binding was affected by factors known to influence explicit binding (when observers must report the explicit relation between the features present). Do the factors that impact on explicit 
binding also affect implicit binding? This would help to provide some constraints on our understanding of implicit binding, and it would be consistent with explicit and implicit binding's sharing common processes.

In Experiment 1, we examined implicit binding in a Stroop color-word task with patient G.K. In Experiment $1 \mathrm{~A}$, we replicated the experiment described by Wojciulik and Kanwisher (1998, Experiment 1). In Experiment $1 \mathrm{~B}$, we carried out the same experiment, but with a larger distance between the colored and the white words. A. Cohen and Ivry (1989) have shown that ICs in normal observers increase when stimuli are spatially close, in comparison with when they are more distant (although see Treisman, 1998), and we replicated this in an explicit binding task with G.K. (Experiment 1C). In Experiment 1D, we repeated the results from Experiment $1 \mathrm{~B}$, but using a within-session manipulation of the distance between the colored and the white words, keeping the white words at fixation, to increase the likelihood that they would be processed. We also used an explicit task in which the names of the colored and white words were color names in order to maximize the probability of binding, since binding may be stronger between semantically related items (color words and colors) than between unrelated items (neutral words and colors, used in the explicit tasks in Experiments 1A-1C). By contrasting performance in Experiment $1 \mathrm{~A}$ with that in Experiments $1 \mathrm{~B}$ and 1D, we assessed whether implicit binding also would be affected by a variable that influences explicit binding (the distance between the stimuli). In Experiment 2, we assessed implicit binding in a spatial variant of the Stroop task. In Experiment 2A, word stimuli were presented at different relative positions within a box (as in Robertson et al., 1997). In Experiment 2B, words were presented at different positions relative to a horizontal line (the horizontal line in the box nearest to the word). In Experiment 2C, as in Experiment 1D, we conducted a within-session manipulation of different binding conditions, contrasting the box and the line conditions (from Experiments $2 \mathrm{~A}$ and $2 \mathrm{~B}$ ) with a further condition in which the words were surrounded by partial boxes (the part-box condition, using the lines from the box other than the one used in the line condition). In Experiment 2D, we showed that explicit binding varied across the different conditions; it was strongest between the words and the box, less strong between the words and the open box, and weakest between the words and the line (note that the words and the box may group on the basis of the principle of common enclosure [Palmer \& Rock, 1994], whereas there are no strong grouping cues to link the word to the line). In Experiment 2, we again asked whether there would be stronger implicit binding between elements that are easier to bind explicitly.

\section{Case Report}

G.K. (born in 1939) suffered two consecutive strokes in 1986, which caused lesions in the right occipito-parietal, right temporo-parietal, and left temporo-parietal regions (see Gilchrist, Humphreys, \& Riddoch, 1996, for the scan). As a consequence of these lesions G.K. manifests Balint's syndrome, whose symptoms include optic ataxia (misreaching under visual guidance), simultanagnosia, and extinction. In everyday life, G.K. manifests great difficulties in the perception of complex multiple object scenes, and he seems to be aware of one object only (or part of it) at a time. He is slightly impaired in word finding, but he has intact memory and comprehension (more details on G.K.'s performance are reported in Cooper \& Humphreys, 2000; Gilchrist et al., 1996; Humphreys et al., 2000; Humphreys, Romani, Olson, Riddoch, \& Duncan, 1994). G.K.'s extinction has been tested in several studies. Humphreys et al. (1994) found that when a picture and a word were presented one above the other, the word was extinguished by the picture. They also found that when two shapes with different degrees of closure were presented together, G.K. selected the more "closed" shape at the expense of the other, showing nonspatial extinction based on object properties. However, under conditions of bilateral presentation, G.K. manifests left-side spatial extinction (Gilchrist et al., 1996). Further experiments have shown that extinction with bilateral presentation is modulated by grouping based on a variety of Gestalt factors (collinearity, common shape, and brightness), as well as by common enclosure (Humphreys, 1998). Testing here was initially conducted between 1999 and 2001 (Experiments 1A-1C, Experiments 2A and 2B), and also in 2003 and 2004 (Experiment $1 \mathrm{D}$ and Experiments $2 \mathrm{C}$ and 2D). G.K.'s performance was stable across this time.

\section{EXPERIMENT 1 Implicit Binding of Color and Shape \\ Experiment 1A Implicit Binding With Close Stimuli}

In Experiment 1, we examined explicit and implicit binding, using a version of the Stroop color-word task in which a colored and a white word were present on every trial (following Wojciulik \& Kanwisher, 1998, Experiment 1). There were again the two critical conditions, SOI and DOI (see Figure 1A). Experiment 1A was a direct replication of Wojciulik and Kanwisher. To test whether G.K. had explicit knowledge of how the features were bound together, each block of the critical color-naming trials (testing implicit binding) was preceded by a block in which G.K. had to perform an explicit decision on word and color binding. In this explicit task, the displays presented were the same as those used in the implicit task, except that neutral words were used instead of color-name words (see Wojciulik \& Kanwisher, 1998). The task was to read the colored word (e.g., given the word HAPPY shown in green and the word READY shown in white, G.K. had to name the word HAPPY). Therefore, in this task, G.K. was forced to explicitly bind the word with the correct color (whereas in the implicit task, this was not required). The presentation of each display was set at the duration at which G.K. had made between $42 \%$ and $54 \%$ correct 
responses in the explicit binding task, at which point we could assume that G.K. was at chance in binding together the word and its color. The same duration was then used for the implicit task.

\section{Method}

Subject. G.K. was the sole subject.

Design and Procedure. In the implicit task, on each trial, two words were presented on a black background. The possible words were BROWN, GREEN, PURPLE, and YELLOW, presented in uppercase Helvetica font, size 48. One word was white, and the other was colored (brown, green, purple, or yellow). The words were presented one above the other, at the center of the screen, separated by a gap of about $0.8 \mathrm{~cm}$, and the colored word was on the upper position on half of the trials. Each word could be either consistent or inconsistent with the color, and as a result, there were four possible conditions: (1) in the both objects inconsistent (BOI) condition, both words were inconsistent with the color; (2) in the same object inconsistent (SOI) condition, the colored word was inconsistent with the color, and the white word was consistent; (3) in the different object inconsistent (DOI) condition, the white word was inconsistent, and the colored word was consistent; and (4) in the neither object inconsistent (NOI) condition, both words were consistent with the color.

Following Wojciulik and Kanwisher (1998), the critical comparison was between the SOI and the DOI conditions. In both conditions, one word was inconsistent with the nonwhite color, but in the SOI condition this was the colored item, whereas in the DOI condition it was the white item. If there is binding of the word with the color, response times (RTs) should be longer in the SOI condition than in the DOI condition. Examples are shown in Figure 1A. All the combinations of colors and words were used, with the constraint that one word had to be white and that the two words were the same only in the NOI condition. The display with the words was preceded by a fixation cross, presented at the center of the screen for $1 \mathrm{sec}$. G.K.'s task was to name the nonwhite color as soon as the words appeared. The RTs were recorded through a microphone. G.K. completed 24 blocks of 96 trials each. However, the first 5 blocks were given in order to familiarize the patient with the task. Each block had an equal number of trials per condition.

The stimuli used in the explicit task were similar to those used in the implicit task. However, in the explicit task, the words were neutral with respect to color. The words were HAPPY, READY, CASUAL, and SHORT (see Figure 1B). As in the implicit task, one word was colored and the other was white, and both were presented against a black background. G.K. had to name the colored word. The purpose of this task was to determine the duration at which G.K. made about $50 \%$ errors (i.e., when he named the white word instead of the colored word). This was interpreted as the patient's being unable to explicitly bind the color with the correct word. Each session started with the presentation of several explicit task blocks (each of 24 trials) during which the duration of the word presentation was gradually adjusted until G.K. ranged between $42 \%$ and $54 \%$ correct. Then an implicit task block was run with that duration. This was followed by another explicit task block. If G.K.'s performance was not within the $42 \%-54 \%$ range, the duration of the word presentation was adjusted again, following the criteria above, before another implicit task block was run. The average duration at which G.K. was at chance level on the explicit task was $240 \mathrm{msec}$ (range, $180-285 \mathrm{msec}$ ).

The stimuli were presented using VSCOPE on a Macintosh IIci machine.

\section{Results and Discussion}

The average RTs and the percentages of correct responses in each condition are shown in Table 1. For each condition, the averages of the median RTs for each block
Table 1

Average Response Times (RTs, in Milliseconds) and Percentage of Correct Responses $(\% \mathrm{C})$ in the Four Conditions in

Experiment 1A (Implicit Binding Task; Close Condition)

\begin{tabular}{|c|c|c|c|c|}
\hline \multirow[b]{3}{*}{ Colored Word } & \multicolumn{4}{|c|}{ White Word } \\
\hline & \multicolumn{2}{|c|}{ Inconsistent } & \multicolumn{2}{|c|}{ Consistent } \\
\hline & RT & $\% \mathrm{C}$ & RT & $\% \mathrm{C}$ \\
\hline Inconsistent & $\begin{array}{r}1,400 \\
\text { (bot } \\
\text { inco }\end{array}$ & $\begin{array}{l}69 \\
\text { ects } \\
\text { ent) }\end{array}$ & $\begin{array}{r}1,261^{*} \\
\text { (sam } \\
\text { incon }\end{array}$ & $\begin{array}{l}74 \\
\text { ect } \\
\text { ent) }\end{array}$ \\
\hline Consistent & $\begin{array}{r}\text { 1,173* } \\
\text { (diffe } \\
\text { inco }\end{array}$ & $\begin{array}{l}81 \\
\text { bject } \\
\text { ent) }\end{array}$ & $\begin{array}{l}1,100 \\
\text { (neith } \\
\text { incon }\end{array}$ & $\begin{array}{r}83 \\
\text { jeect } \\
\text { ent) }\end{array}$ \\
\hline
\end{tabular}

*The two critical conditions.

were calculated (incorrect responses were not included; see Wojciulik \& Kanwisher, 1998). A two-way withinsubjects design ANOVA was used to analyze the data, treating block as the random factor. There was a reliable main effect of whether the name of the colored word was consistent or inconsistent with its color $[F(1,18)=28.82$, $p<.001]$ and also a reliable effect of the consistency of the name of the white word and the color of the colored word $[F(1,18)=12.30, p<.01]$. The interaction was not reliable $[F(1,18)=1.23, p>.05]$. The critical comparison was between the SOI and DOI conditions. RTs in the SOI condition $(1,261 \mathrm{msec})$ were significantly longer than those in the DOI condition $(1,173 \mathrm{msec})[t(18)=2.58$, $p=.019]$. These results suggest that even when G.K. was at chance in binding the color with the correct word, the correct binding was nonetheless processed by his visual system at some (preattentive) level, even if he was not aware of it. Accordingly, there was more interference when the color was inconsistent with (and bound to) the colored word than when it was inconsistent with (and bound to) the white word. This held across both RTs and errors.

G.K.'s accuracy data were analyzed using a threefactor log-linear analysis (white word consistency, colored word consistency, correct/error). There was one reliable interaction between colored word consistency and accuracy $\left[\chi^{2}(1)=28.97, p<.001\right]$. There were proportionately more errors when the name of the colored word was inconsistent with its color than when it was consistent.

ICs have often been considered as evidence that there is no spatial information available when stimuli are not attended and that attention is necessary both for detecting the spatial location of the features perceived and for feature binding (Treisman, 1998; Treisman \& Schmidt, 1982). However, other studies have shown that ICs are affected by the distance between the stimuli (the closer the stimuli, the more frequent the ICs). This has led to the conclusion that spatial processing, even if not precise, is possible at the preattentive stages (A. Cohen \& Ivry, 1989; A. Cohen \& Rafal, 1991). The results of Experiment 1A (and prior findings on implicit binding) show that spatial processing at preattentive levels of visual perception can be precise enough to bind properly object features. There are various ways to interpret these data on implicit binding. One possibility is that explicit and implicit binding 
of visual features are two qualitatively distinct processes, perhaps operating at different levels of processing. $\mathrm{Pa}-$ tients with parietal damage, such as G.K., are impaired at the high-level binding process required for conscious report. They are not impaired at a lower level binding process, as revealed in implicit judgments. In other words, implicit binding may be intact. A somewhat different account is that binding is graded and not an all-or-none process. The strength of binding may change as a function of various factors, including the processing time over which binding is critical for the response and whether attention can be brought to bear on the stimuli. The effect of attention may be to increase the strength of binding, stabilizing a given binding relationship. In addition, without attention, the strength of binding may decrease across time. So, when a time-consuming conscious judgment about the relations between visual features must be made and a patient is impaired at attending to the stimulus, the features may become unbound across time. In contrast, there may be a much shorter time over which it is critical that a word and a color are bound in order to generate Stroop interference. Over this shorter time period, the features may be bound even without attention (e.g., by co-occurring at the same location). It follows that a Stroop effect could emerge when the color and the incongruent word are in the same location, even when conscious judgments about color-form binding are at chance. In relation to these comments, it is interesting to note that G.K. frequently remarked that he felt that he performed better if he could make a response based on his "first glance" at a stimulus and that, often, the more he looked at stimuli the more he became confused, with one element being linked (incorrectly) to others.

To further study the relations between implicit and explicit binding, in Experiments $1 \mathrm{~B}-1 \mathrm{D}$, we investigated whether the spatial distance between stimuli would influence both implicit binding (Experiments 1B-1D) and explicit binding (Experiment 1C).

\section{Experiment 1B \\ Implicit Binding With Distant Stimuli}

The purpose of Experiment 1B was to see whether the implicit binding in G.K., found in Experiment 1A, would be affected by the distance between the stimuli. This experiment was very similar to Experiment $1 \mathrm{~A}$, with the only difference being that the colored and the white word presented on each trial were more distant from each other.

\section{Method}

As in Experiment 1A, G.K. was presented with explicit task blocks followed by implicit task blocks. In both cases, the stimuli and procedure were identical to those used in Experiment 1A. The only difference was that the two words presented were more distant than before $(3.5 \mathrm{~cm})$. G.K. completed 19 task blocks of 48 trials each, with each block containing a set of explicit and implicit binding trials. Performance was set to chance in the explicit binding trials, with the average duration being $827 \mathrm{msec}$. This long duration, relative to the duration used in Experiment 1A, likely reflected the

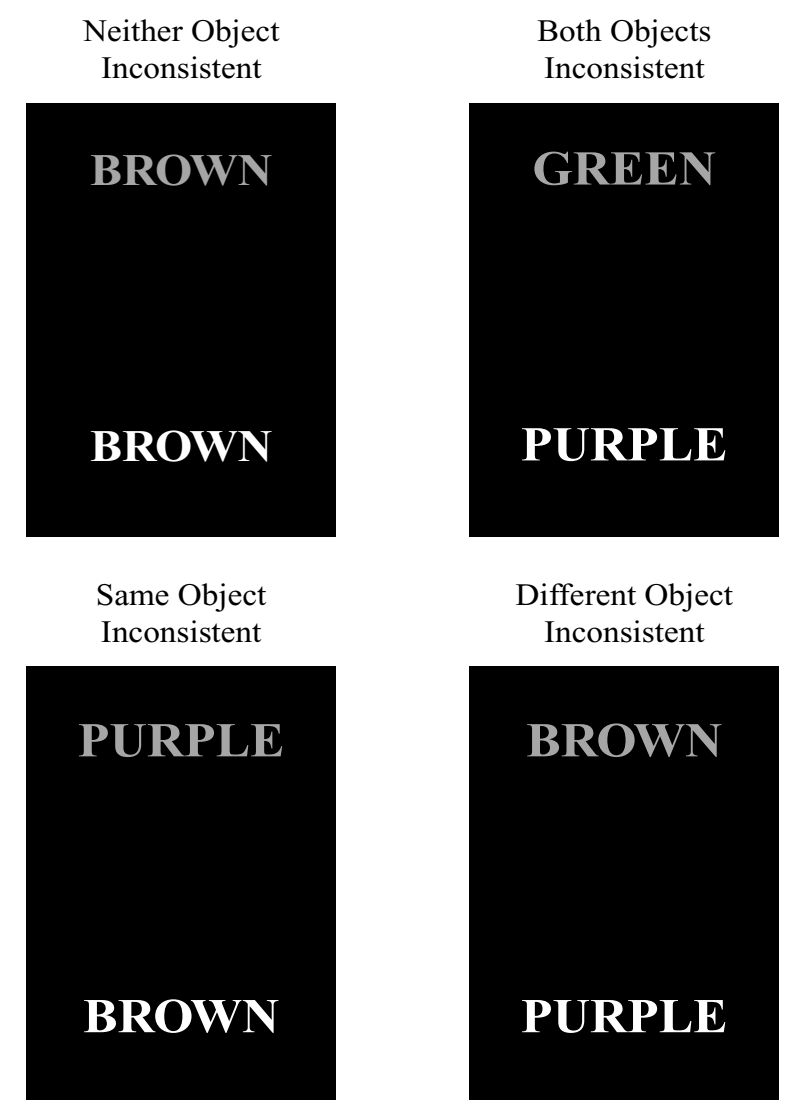

Figure 2. Examples of the displays used in the implicit binding conditions in Experiment $1 B$ (the task was to name the color). There were equivalent separations between the words in the explicit binding condition (where neutral words were used).

spatial locations at which the stimuli appeared, since the stimuli were closer to fixation in Experiment $1 \mathrm{~A}$ than in Experiment 1B. Example displays are shown in Figure 2.

\section{Results and Discussion}

The average RTs and the percentages of correct responses for each condition are shown in Table 2. A twofactor within-subjects ANOVA, with block as the random factor, revealed a main effect of the color consistency of the color word $[F(1,18)=20.46, p<.001]$. However, there was no main effect of the consistency of the white word $(F<1)$ and no interaction $(F<1)$. The difference between the RTs in the two critical conditions, SOI (1,731 msec) and DOI (1,357 $\mathrm{msec})$, was significant $[t(18)=3.01, p=.007]$, suggesting that there was implicit binding. To see whether the difference between the SOI and the DOI conditions was modulated by the distance between the two words, a two-way ANOVA was performed to compare the difference between the two conditions in this experiment and Experiment $1 \mathrm{~A}$. The two factors were distance (the stimuli were close in Experiment $1 \mathrm{~A}$ and more distant in Experiment 1B) and consistency (SOI 
Table 2

Average Response Times (RTs, in Milliseconds) and Percentage of Correct Responses $(\% \mathrm{C})$ in the Four Conditions in

Experiment 1B (Implicit Binding Task; Distant Condition)

\begin{tabular}{|c|c|c|c|c|}
\hline \multirow[b]{3}{*}{ Colored Word } & \multicolumn{4}{|c|}{ White Word } \\
\hline & \multicolumn{2}{|c|}{ Inconsistent } & \multicolumn{2}{|c|}{ Consistent } \\
\hline & RT & $\% \mathrm{C}$ & RT & $\% \mathrm{C}$ \\
\hline \multirow[t]{2}{*}{ Inconsistent } & 1,675 & 68 & $1,731^{*}$ & 73 \\
\hline & $\begin{array}{l}\text { (botl } \\
\text { inco }\end{array}$ & & $\begin{array}{l}\text { (sam } \\
\text { incol }\end{array}$ & $\begin{array}{l}\text { ect } \\
\text { nt) }\end{array}$ \\
\hline \multirow[t]{2}{*}{ Consistent } & $1,356^{*}$ & 74 & 1,338 & 82 \\
\hline & $\begin{array}{r}\text { (differ } \\
\text { inco }\end{array}$ & $\begin{array}{l}\text { ject } \\
\text { ht) }\end{array}$ & $\begin{array}{l}\text { (neith } \\
\text { incol }\end{array}$ & $\begin{array}{l}\text { ject } \\
\text { nt) }\end{array}$ \\
\hline
\end{tabular}

*The two critical conditions.

condition vs. DOI condition). There was a main effect of distance $[F(18)=18.08, p<.001]$ : Overall, G.K. was slower when the two words were more distant. This is not surprising, given that the target stimuli fell further from fixation in Experiment 1B. The main effect of consistency was also significant $[F(18)=13.47, p=.002]$ : G.K. was slower when the hue of the colored word was inconsistent with its name (in the SOI condition vs. the DOI condition). Finally, there was a significant interaction between the two factors $[F(1,18)=4.73, p=.043]$ : The difference between the two conditions was smaller when the words were closer $(\mathrm{SOI}-\mathrm{DOI}=88 \mathrm{msec})$ than when they were more distant $($ SOI - DOI $=375 \mathrm{msec})$. This difference might have been due to the fact that on average, RTs in Experiment 1B were much longer than RTs in Experiment 1A, presumably reflecting the more peripheral locations of the stimuli. However, even when scaled by the absolute RTs, the ratio of the lengthening of RTs in the SOI condition was greater than that in the DOI condition in Experiment 1B, relative to Experiment $1 \mathrm{~A}(1.29$ vs. 1.07).

A three-factor log-linear analysis of G.K.'s errors with the distant stimuli revealed two interactions, between consistency of the white word and accuracy $\left[\chi^{2}(1)=5.19\right.$, $p<.05]$ and between consistency of the colored word and accuracy $\left[\chi^{2}(1)=6.66, p<.05\right]$. Hence, although there was no effect of the consistency of the white word on RTs, there was an effect on errors. Performance was relatively more accurate when the name of the white word was consistent with the response (to the colored word) than when it was inconsistent. As in Experiment 1A, the consistency between the name and the color of the colored word also influenced response accuracy, as well as RTs.

How can we explain the finding that at least for RTs, the difference between the critical Stroop conditions was smaller in Experiment 1A (close distance) than in Experiment 1B (distant stimuli)? We attribute the change in Stroop effects to the involvement of ICs under the present conditions and to the quality of perceptual information available when ICs occur. Consider what might happen if ICs sometimes occur under the present conditions, when two words are presented in two different colors. An IC in the SOI condition would reduce Stoop interference. For instance, the words BROWN (in purple) and PURPLE (in white) might be coded as PURPLE (in purple) and BROWN (in white). This should facilitate performance. However, any beneficial effect in this condition would be traded off against an increase in Stroop interference produced by an IC in the DOI condition. Then the words PURPLE (in purple) and BROWN (in white) might be coded as PURPLE (in white) and BROWN (in purple). Performance should then be slowed. Since there is no reason to expect ICs to be more frequent in the SOI condition, there should be a neutral relationship between ICs and Stroop interference. However, a different picture would emerge if ICs are associated with relatively "weak" perceptual representations, in which (for example) the features are not well coded. This is not unreasonable. On many accounts (e.g., Treisman, 1998), ICs should arise on trials in which the locations of features are poorly represented, and this could particularly disrupt word representations where the locations of the letters are critical to the word's identity. Indeed, prior research suggests that in addition to form-color exchanges taking place, there can be exchanges of letters between words when attention to the words is disrupted (McClelland \& Mozer, 1986). G.K. makes such errors in his reading even under prolonged viewing conditions (Hall, Humphreys, \& Cooper, 2001), and they may be even more likely to occur when viewing times are reduced. The net result of this would be that word representations are likely to be less well coded on trials in which ICs occur. Given the standard asymmetry of Stroop interference, in which costs on incongruent trials are larger than the benefits present when the word and its color are congruent (J. D. Cohen, Dunbar, \& McClelland, 1990), we would expect Stroop interference on incongruent trials, in particular, to be reduced when word identities are poorly coded (when ICs occur). It follows that Stroop interference will be larger when the stimuli are distant, relative to when they fall at neighboring locations, if distance reduces the likelihood that ICs will occur.

One puzzle, though, is why there was no effect of the consistency of the white word on RTs in this experiment, given that there was an effect of the consistency of the colored word (although note that there was some effect on errors). The reduced effect of the white word can be attributed to its falling at a location more distant from the colored word and at a more peripheral location than in Experiment 1A, so that the meaning of the white word had less impact on response selection. Yet despite this, G.K. was still at chance at naming the colored word when we tested his explicit knowledge of the binding relation between the nonwhite color and the word. If the white word had less impact in Experiment 1B, we would expect G.K. to have benefited on the test of explicit binding. However, the test of explicit binding could itself have suffered from the more peripheral presentation of the words in Experiment 1B, so that G.K. on some occasions had less accurate information about the word identities per se (especially when the exposure duration was limited). That is, errors on the explicit binding task could reflect misidentifications of the colored word, and not simply incorrect bind- 
ings between the words and their colors. This would be consistent with our proposal that ICs were less likely to occur in Experiment 1B than in Experiment 1A.

Experiment $1 \mathrm{C}$ was conducted to test this last proposal more directly, using the stimuli from Experiments $1 \mathrm{~A}$ and 1B. In Experiment 1C, the word displays from the explicit binding task were presented for unlimited durations, and G.K. was asked to report the words and their colors. We assessed whether ICs were greater at the close than at the more distant separation.

\section{Experiment 1C Explicit Report Under Unlimited Conditions Method}

The stimuli were the same as those in the explicit binding conditions in Experiments 1A and 1B. The stimuli were the words HAPPY, READY, CASUAL, and SHORT. Two words appeared on each trial, one above and one below fixation, separated by either 0.8 or $3.5 \mathrm{~cm}$. One word was always presented in white, and the other was brown, green, purple, or yellow. There were 192 trials at each separation (12 combinations of words $\times 16$ color arrangements), presented in a fully random order in two separate trial blocks. G.K. was asked to name both the words and their colors. The stimuli appeared for an unlimited duration, preceded by a central fixation cross for $1 \mathrm{sec}$. Stimulus presentations were controlled by E-Prime, and the experiment was run using a Pentium PC.

\section{Results and Discussion}

G.K. named both the words and their colors on 120/192 $(64 \%)$ of the trials at the close separation and 155/192 $(81 \%)$ of the trials at the distant separation. Performance was better when the stimuli were more distant than when they were close to one another (even though the more distant items fell at more peripheral retinal locations) $\left[\chi^{2}(1)=14.81, p<.01\right]$. G.K. made 41 IC responses when the stimuli were close (on $21 \%$ of the trials), where he reported one of the words in the color of the other word present ( 22 of these trials involved complete feature exchanges, where each word was reported in the color of the other word). On the remaining 31 error trials, he misnamed either one of the words or one of the colors, generating a response that was unrelated to either of the stimuli present. In the distant condition, G.K. made 11 IC errors (two complete feature exchanges) and 26 unrelated errors. Relative to the number of unrelated errors, G.K. made proportionately more ICs when the words were close than when they were distant $\left[\chi^{2}(1)=6.21, p<.025\right]$.

These results confirm those of previous studies of ICs in normal observers, where at least some investigators have reported that ICs decrease as a function of the distance between stimuli (A. Cohen \& Ivry, 1989). Here, G.K. generated proportionately fewer ICs when the words were spatially separated than when they were spatially close, although unlike controls, G.K. made ICs with unlimited stimulus exposures. This again indicates his problem in explicitly binding shape and color (see also Humphreys et al., 2000). The results are consistent with the proposal that ICs, between close stimuli, could have contributed to the Stroop effects we observed in Experiment 1A. The data also demonstrate that G.K.'s performance on a task requiring explicit binding of shape and color was affected by a variable common to his implicit binding (in Experiments $1 \mathrm{~A}$ and $1 \mathrm{~B}$ ).

Although the data from Experiments $1 \mathrm{~B}$ and $1 \mathrm{C}$ are consistent with the proposal that G.K. showed a stronger Stroop effect with distant stimuli because there was less chance of a binding error between the two words, the case for a relation between implicit and explicit binding is weakened by the likelihood that the white word may not have been well processed in the implicit binding task. Implicit and explicit binding could still be independent processes, but both could be affected by a common data limitation when the words are presented more peripherally and under reduced exposure durations (in Experiment 1B). The evidence for common processes in implicit and explicit binding would be stronger if we could show both a stronger Stroop effect and reduced explicit binding under conditions in which the white word was more likely to be processed. This was the aim of Experiment 1D. As in Experiments 1A and 1B, we included tests of explicit binding along with tests of implicit binding. However, in Experiment 1D, we used color names, rather than neutral words, for the measures of explicit binding. It is possible that binding can be stronger between semantically related items (color words and colors) than between unrelated items (neutral words and colors; see Kornblum \& Stevens, 2002). If this held, the apparent better implicit than explicit binding in Experiments 1A and 1B could be due to the contrast between the stimuli used, and not to the implicit and explicit measures per se. This argument is not viable when colored words are used in both procedures. Finally, as in Experiment 1C, we also ran a final session in which G.K. was asked to name the words and the colors under unlimited presentation conditions, in order to gain a measure of IC errors under these presentation conditions.

\section{Experiment 1D \\ Implicit and Explicit Binding With the White Word at Fixation}

\section{Method}

The stimuli were the same size and presented in the same font as in Experiment 1A, and they were presented using E-Prime. However, in this case, the white word was always presented at fixation, and the colored word appeared randomly above or below fixation, separated by either $0.8 \mathrm{~cm}$ (as in Experiment $1 \mathrm{~A}$ ) or $3.5 \mathrm{~cm}$ (as in Experiment 1B). There were again four colors and four color words, but here the colors were brown, green, yellow, and blue, which, on implicit binding trials, were used along with their corresponding words (BROWN, GREEN, YELLOW, and BLUE). The word and color blue were substituted here for purple because we now employed a set of different color words in the explicit binding trials (rather than neutral words) and wanted to match the length of the words to those used in the implicit binding trials. For the explicit binding trials, the words were BLACK, CLARET, ORANGE, and GRAY. By using different color words to the colors present on explicit binding trials, we ensured that we could tell whether G.K. was reporting a color or the word when he was asked to read the colored word on a given trial. The white word was paired with each of the other colors equally often, and in the implicit binding task, the words were in each of the 
four conditions equally often (BOI, SOI, DOI, and NOI). Each trial block was 256 trials long, giving 32 presentations in each condition at each separation (pooling across trials when the colored word fell above and when it fell below fixation). At least 2 blocks of explicit binding trials, with 64 trials, was run before each block of implicit binding trials, and the duration of the stimuli was adjusted until G.K. scored about $50 \%$ at reading the colored word. Across the trial blocks, G.K. identified $54 \%$ and $48 \%$ of the colored words correctly at the close and far separations, respectively. Performance in each case did not differ from chance $\left(\chi^{2}<1\right)$. The mean duration across the trial blocks was $550 \mathrm{msec}$. This duration was longer than that used in Experiment 1A, most likely because the colored word could fall further from fixation here on half the trials; nevertheless, it was shorter than that used in Experiment 1B, perhaps because spatial uncertainty was reduced, with the white word always appearing at fixation, and perhaps also because G.K. found the color words easier than the neutral words in the explicit binding trials. There were 14 blocks of trials.

\section{Results and Discussion}

The means across blocks of the median correct RTs in each block, along with the percentages of correct responses, are given in Table 3 . The results were analyzed using a three-factor repeated measures ANOVA (distance, consistency of white word, and consistency of colored word), with block as a random factor. There were reliable main effects of distance $[F(1,13)=143.21, p<.001]$, consistency of the white word $[F(1,13)=23.83, p<.001]$, and consistency of the colored word $[F(1,13)=82.84, p<$ $.001]$. There were two reliable interactions: between distance and the consistency of the colored word $[F(1,13)=$ $4.72, p<.05]$ and between the consistency of the white word and the consistency of the colored word $[F(1,13)=$ $16.32, p<.001]$. The critical effects, in the SOI and DOI conditions, were examined in a two-factor ANOVA with condition (SOI vs. DOI) and distance as the factors. There were main effects of distance $[F(1,13)=140.54, p<$

Table 3

Average Response Times (RTs, in Milliseconds) and Percentages of Correct Responses (\%C) in the Conditions in Experiment 1D, for Colored Words Either Close to or Distant From the White Word at Fixation

\begin{tabular}{|c|c|c|}
\hline \multirow[b]{3}{*}{ Colored Word } & \multicolumn{2}{|c|}{ White Word } \\
\hline & Inconsistent & Consistent \\
\hline & RT $\% \mathrm{C}$ & RT $\quad \% \mathrm{C}$ \\
\hline \multicolumn{3}{|c|}{ Close Words } \\
\hline Inconsistent & $\begin{array}{l}1,649 \quad 68 \\
\text { (both objects } \\
\text { inconsistent) }\end{array}$ & $\begin{array}{l}1,464^{*} \\
\text { (same object } \\
\text { inconsistent) }\end{array}$ \\
\hline Consistent & $\begin{array}{l}1,331^{*} \\
\text { (different object } \\
\text { inconsistent) }\end{array}$ & $\begin{array}{c}1,046 \quad 82 \\
\text { (neither object } \\
\text { inconsistent) }\end{array}$ \\
\hline \multicolumn{3}{|c|}{ Distant Words } \\
\hline Inconsistent & $\begin{array}{c}1,960 \quad 62 \\
\text { (both objects } \\
\text { inconsistent) }\end{array}$ & $\begin{array}{c}1,932^{*} \\
\text { (same object } \\
\text { inconsistent) }\end{array}$ \\
\hline Consistent & $\begin{array}{l}1,630^{*} \\
\text { (different object } \\
\text { inconsistent) }\end{array}$ & $\begin{array}{l}1,433 \\
\text { (neither object } \\
\text { inconsistent) }\end{array}$ \\
\hline
\end{tabular}

*The two critical conditions.
$.001]$ and condition $[F(1,13)=37.90, p<.001]$ and $a$ distance $\times$ condition interaction $[F(1,13)=11.97, p<$ $.01]$. RTs were longer when the colored word fell further from fixation; they were longer in the SOI than in the DOI condition (i.e., there was an effect of implicit binding), and the difference between the conditions increased when the colored word was more distant from the central white word (an effect of $143 \mathrm{msec}$ when the words were close vs. $302 \mathrm{msec}$ when they were distant). However, the condition effect was reliable at both distances $[t(13)=-3.08$ and -6.24 , both $p s<.01$, for the close and more distant separations, respectively].

The error data followed the same pattern as the RT data. A four-factor log-linear analysis was undertaken, with the factors being distance, consistency of the white word, consistency of the colored word, and accuracy (correct or error). There were reliable interactions between distance and accuracy, between consistency of the white word and accuracy, and between consistency of the colored word and accuracy $\left[\chi^{2}(1)=13.24,31.07\right.$, and 13.24, all $p \mathrm{~s}<$ $.001]$. G.K.'s accuracy was reduced when the colored word fell further from fixation, when the white word was inconsistent with the color of the colored word, and when the name of the colored word was inconsistent with its color.

These data essentially replicate the findings from Experiments $1 \mathrm{~A}$ and $1 \mathrm{~B}$. RTs were longer and accuracy was lower when the name of the colored word was inconsistent with its color (SOI condition) than when the name of the white word was inconsistent with the colored word (but the name of the colored word was consistent; DOI condition). This demonstrates an implicit binding effect, since performance was set at a duration at which explicit binding was at chance. As before, the size of the difference between the conditions increased as the distance between the white and the colored words increased. We again attribute this to the tendency of ICs to be reduced as the words fell at more distant locations. To provide a direct test of this, we reran one session, using the stimuli from the explicit binding task but using unlimited stimulus exposures. There were two blocks of 64 trials. G.K. was asked to name both the words and their colors, and performance was scored according to whether each word was correctly paired with its color. He named both the words and their colors correctly on $39 / 64(61 \%)$ of the trials when the stimuli were close and on 54/64 (84\%) of the trials when they were distant. Performance was better when the stimuli were more distant $\left[\chi^{2}(1)=9.30, p<.01\right]$. When the words were close, G.K. made $12 / 25$ (48\%) IC errors; when the words were distant, he made just $1 / 10(10 \%)$ IC errors. There was a higher rate of ICs when the stimuli were close than when they were more distant (Fisher exact probability $=.039$, one-tailed). ICs were reduced when the words were more distant. We suggest that since ICs were reduced at the wider separation, there would be a reduced likelihood of incorrect bindings in the implicit binding condition. As a consequence, the effect of the colored word's being incongruent with its color (in the SOI condition) increased. Unlike in Experiment 1B, when both words appeared away 
from fixation, there was evidence here that the white word was processed: Performance was disrupted when the identity of the white word was inconsistent with the nonwhite color. Hence, the experiment establishes that distance affects both explicit and implicit binding under conditions in which the white word was processed and was not subject to data limitations.

It is also interesting to note that the evidence for implicit binding here, when explicit binding was at chance, arose even though both the implicit and the explicit binding tasks involved colors and names for color words; indeed, if anything, G.K. made a higher rate of explicit ICs under unlimited stimulus exposures here than when neutral words were used (48\% vs. $21 \%$ when the stimuli were close). Hence, the difference between implicit and explicit binding cannot simply be attributed to there being stronger binding between semantically related items in the earlier experiments.

\section{EXPERIMENT 2 Implicit Binding of Forms}

In Experiment 1, we found evidence for implicit binding of shape and color, even when G.K. could not explicitly identify the shape and color that went together. Implicit binding was also affected by a variable that influenced explicit binding of color and shape - that is, spatial distance. In Experiment 2, we examined the binding of form elements, rather than the binding of form and color. In Experiment 2A, we carried out an experiment similar to that described by Robertson et al. (1997). G.K. was presented with a vertical rectangle that contained, either at the top or at the bottom, a word (UP or DOWN) that could be either consistent or inconsistent with its position inside the box (see Figure 3A). For example, the word UP was consistent with the position when it was at the top of the box, and it was inconsistent when it was at the bottom. G.K.'s task was to read the word as soon as it appeared. If there was implicit binding of the word with its position, we would expect G.K. to be slower when the words were inconsistent with their position. As in Experiments $1 \mathrm{~A}$ and $1 \mathrm{~B}, \mathrm{G} . \mathrm{K}$. also had to perform an explicit task, where he had to explicitly report the position of the word inside the box. The neutral words (i.e., with meanings not related to their position) WELL and GOOD were used, instead of the words presented in the implicit task. Again, the duration of the displays in the explicit task was chosen so that G.K. performed at between $42 \%$ and $54 \%$ correct on reports of word position. Subsequently, the same duration was used for the implicit task.

In Experiment 2B, we used a similar procedure, but this time presented the words UP and DOWN (in the implicit binding task) or WELL and GOOD (in the explicit binding task) above or below a horizontal line. In the implicit binding condition, the word could be consistent with the line (UP above the line, DOWN below the line), or it could be inconsistent with the line (UP below the line, DOWN above the line). In prior studies with G.K., we have shown that he is sensitive to grouping between form elements
A) IMPLICIT TASK

Consistent
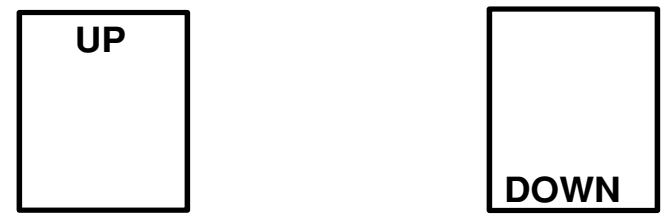

Inconsistent
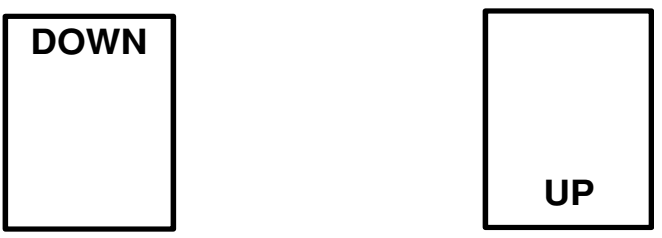

B) EXPLICIT TASK

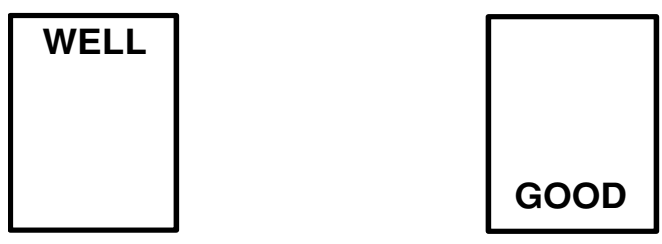

Figure 3. Examples of the displays in Experiment 2A. (A) The consistent and the inconsistent conditions in the implicit task (the task was to read the word). (B) In the explicit task, the words WELL and GOOD were used, instead of the words UP and DOWN (the task was to name the position of the word).

and, in particular, to common enclosure (see Humphreys, 1998). As a consequence, we might expect binding to be stronger between the word and the enclosing box (in Experiment 2A) than between the word and the line (which do not group on the basis of any Gestalt properties save proximity, in Experiment 2B). This was confirmed in Experiment 2D. We asked whether evidence for implicit binding would be stronger with the forms that show stronger explicit binding (with boxes, in Experiment 2A, rather than with the lines, in Experiment $2 \mathrm{~B}$ ). Experiment $2 \mathrm{C}$ extended Experiments $2 \mathrm{~A}$ and $2 \mathrm{~B}$, but using a withinsession manipulation of three grouping conditions: word in a box, word above or below a line (as in Experiments $2 \mathrm{~A}$ and $2 \mathrm{~B}$ ), and word in a three-sided figure (the part-box-i.e., the lines remaining from the box when the line used in Experiment 2B was extracted). Furthermore, in the explicit binding condition in this experiment, we used the same words as those in the implicit binding condition, to ensure that any advantage for implicit binding 
did not occur because, in the explicit binding task, neutral words were used, instead of words related to the relative spatial locations of the stimuli in the displays. Finally, Experiment 2D repeated Experiment $2 \mathrm{C}$ but presented the stimuli for an unlimited duration. G.K. was then required to read the word and to describe its spatial relation to the geometric figure (the box, the line, or the part-box). We evaluated whether problems in explicit binding mirrored differences between the implicit binding conditions.

\section{Experiment 2A \\ Binding Words in Boxes}

\section{Method}

Implicit task. Each trial started with the presentation of a fixation cross, positioned at the center of the screen, for about $1 \mathrm{sec}$. This was followed by the presentation of a word inside a "box" that was a vertical rectangular frame that surrounded the word. The word could be either UP or DOWN; it was black, written in uppercase Helvetica, of size 36 . The frame was black and 8 points thick, and its size was $4.3 \times 6.5 \mathrm{~cm}$. The word was always presented at the center of the screen, but the box had two possible positions, so that the word appeared to be either at the top of the box (when the box was on the lower position) or at the bottom (when the box was on the upper position; see Figure 3A). The distance between the word and the box's segment closest to the word was $0.7 \mathrm{~cm}$. Combining words and positions, there were two conditions. In one condition, the word was consistent with its position inside the box, and in the other condition, the word was inconsistent with its position. The task was to read the word as soon as it appeared. RTs were recorded through a microphone. G.K. was presented with 28 blocks of 40 trials each (with same number of trials in each condition). The first 10 blocks were given as practice, and only the remaining 18 blocks were included in the statistical analysis. For each block, only the trials with the correct response were analyzed.

Explicit task. The stimuli used were similar to those used for the implicit task; however, this time, words neutral to the positions were employed (GOOD and WELL). The words were presented inside the box (see Figure 3B), and the task of the patient was to name the position of the word, relative to the box. The aim of this task was to find the stimulus presentation duration at which G.K. was at chance in binding the word with its position. To do that, the procedure was the same as that used in Experiment 1: Once the duration at which G.K.'s performance ranged between $42 \%$ and $54 \%$ correct was found, two implicit task blocks were run, followed by one or more explicit task blocks. The average presentation duration at which G.K.'s performance was at chance level was $1,200 \mathrm{msec}$ (range, 750-1,600 $\mathrm{msec}$ ).

\section{Results and Discussion}

The results of Experiment $2 \mathrm{~A}$ were divided into two conditions. In the consistent condition, the word UP was presented at the top of the box, and the word DOWN was presented at the bottom. In the inconsistent condition, the word DOWN was presented at the top of the box, and the word UP was presented at the bottom. In each block, the medians of the RTs of each condition were calculated, and then the averages of the medians of each condition were calculated. The average RTs were shorter in the consistent condition $(1,188 \mathrm{msec} ; 80.4 \%$ correct $)$ than in the inconsistent condition $(1,261 \mathrm{msec} ; 85.7 \%$ correct). The data were analyzed with a paired-sample $t$ test, where blocks were treated as subjects. The difference between the two conditions was significant $[t(17)=3.17, p=.006]$. The mean number of errors did go in the opposite direction, with fewer errors on inconsistent than on consistent trials; however, this was not significant $\left(\chi^{2}<1\right)$. These data suggest that even though G.K. was at chance when he had to report explicitly the location of the word, in the implicit task there was evidence of correct binding of the word with its relative position in the box. This caused G.K. to be slower when the position of the word was inconsistent with its name. The data illustrate that there is not only implicit binding of form and color (Experiment 1), but also implicit binding of form elements. Experiment 2B was performed to assess whether implicit spatial binding would also occur between a word and a neighboring horizontal line.

\section{Experiment 2B Binding Words and Lines}

Experiment 2B was very similar to Experiment 2A, the only difference being that here, a line was used instead of the box. Each word could be above or below the line, so that its name was equally consistent or inconsistent with its spatial position with respect to the line (see Figure 4). In the implicit task, G.K. had to read the word, whereas in the explicit task, he had to identify the word's position relative to the line (either up or down).

\section{Method}

Implicit task. On each trial, the word UP or DOwN was presented above or below a horizontal black line. The line was $4.3 \mathrm{~cm}$ long, and it was 8 points thick. The distance between the word and the line was

\section{A) IMPLICIT TASK}

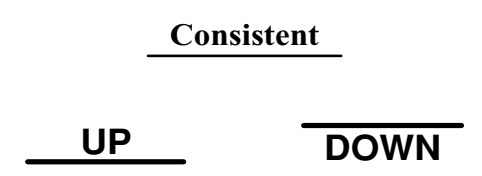

Inconsistent

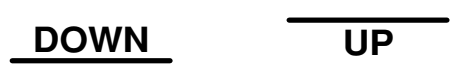

B) EXPLICIT TASK

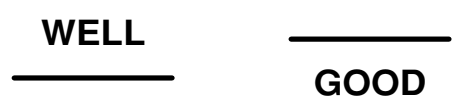

Figure 4. Examples of the displays in Experiment 2B. (A) The consistent and the inconsistent conditions in the implicit task (the task was to read the word). (B) In the explicit task, the words WELL and GOOD were used, instead of the words UP and DOWN (the task was to name the position of the word). 
$0.7 \mathrm{~cm}$. The word was always presented at the center of the screen. In the consistent condition, the word UP was above the line, and the word DOwN was below the line (i.e., the name of the word and its position were consistent). In the inconsistent condition, the word UP was below the line, and the word Down was above the line (i.e., the name of the word and its position were inconsistent). The procedure was identical to that used in Experiment 2A. G.K.'s task was to read the word as soon as it appeared. The word and the line were preceded by a fixation cross presented for $1 \mathrm{sec}$.

G.K. was presented first with 5 blocks of practice and then with 19 experimental blocks of 40 trials each (20 trials in each condition).

Explicit task. Either the word GOOD or the word WELL was presented on a trial, above or below the line. G.K. had to name the position of the word (using the words "up" or "down"). The duration at which G.K. scored around $50 \%$ correct was selected, following the same procedure as that in Experiment 2A. The average of that duration was $850 \mathrm{msec}$.

\section{Results and Discussion}

The mean of the correct RTs in the consistent condition was $988 \mathrm{msec}$ (79.7\% correct), whereas it was $961 \mathrm{msec}$ (84.5\% correct) for the inconsistent condition. A pairedsample $t$ test (where blocks were treated as subjects) failed to show any sign of a significant difference in RTs between the two conditions $[t(18)<1]$. There was also no reliable effect on accuracy $\left(\chi^{2}<1\right)$.

Unlike in Experiment $2 \mathrm{~A}$, the present results gave no evidence of implicit binding: Not only was G.K. not able to make explicit judgments concerning the relative position of the word to the line, but also there was no effect of consistency of the line with the word on G.K.'s naming of the word. The results are consistent with implicit spatial binding in Experiment 2A being contingent on grouping of the word and the box by common enclosure; when the grouping cues were reduced (with the horizontal line, rather than with the box), implicit spatial binding apparently did not occur. It is of some interest that Humphreys (1998) also reported that extinction in G.K. was similarly influenced by grouping by common enclosure.

Experiment $2 \mathrm{C}$ extended Experiments $2 \mathrm{~A}$ and $2 \mathrm{~B}$ by including the same binding conditions and adding a third in which we presented the words in part-boxes formed from the lines of the box remaining after the line used in Experiment 2B had been removed.

\section{Experiment 2C \\ Implicit Binding of Words, Boxes, Lines, and Part-Boxes}

\section{Method}

The method was the same as that used in Experiments $2 \mathrm{~A}$ and $2 \mathrm{~B}$, except that three grouping conditions were included: the words (UP or DOWN) appeared in the box (as in Experiment 2A), above or below the line (as in Experiment 2B), or in a part-box (see Figure 5). These conditions occurred equally often and were randomly mixed within a block of 120 trials (40 per condition). In each condition, the position of the word was equally often consistent or inconsistent with regard to its spatial location in relation to its local shape context (the box, the line, or the part-box). In the explicit condition in this experiment, we presented the same stimuli as those in the implicit binding task but required G.K. to name the word and to describe its relation to its local context, using the terms at the top or at the bottom (of the box, the line, or the part-box). There were at least 2

\section{IMPLICIT and EXPLICIT TASKS}
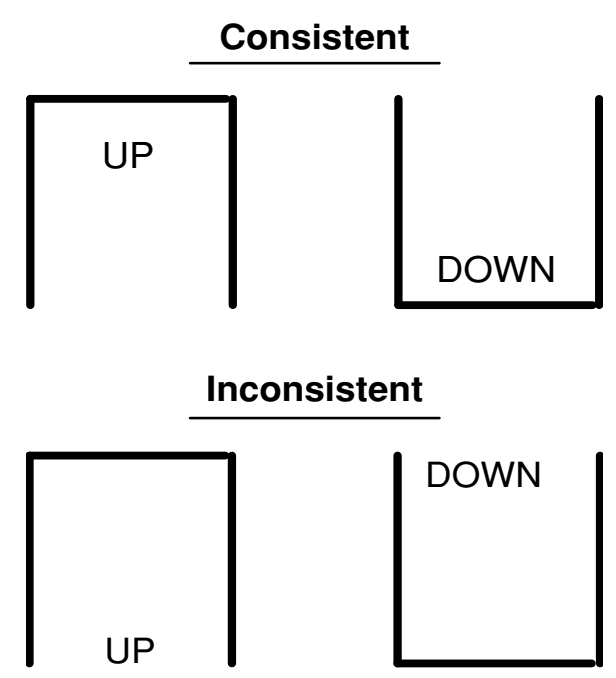

Figure 5. Examples of the part-box displays in Experiment 2C. In this experiment, the same stimuli were used for both the implicit and the explicit tasks. In the explicit task, the task was to name the position of the word in relation to its context (in a full box, in a part-box [as here], or in relation to a line [as in Experiment 2B]). In the implicit condition, the task was to read the word.

blocks of 30 explicit binding trials ( 10 per condition, 5 consistent and 5 inconsistent) prior to each implicit binding block, and we adjusted the durations of the displays until G.K. scored around $50 \%$ correct in the explicit binding trials. This duration was then used for the ensuing implicit binding block. There were 12 blocks of implicit binding trials, and the mean duration across these blocks, derived from the explicit binding trials, was $1,100 \mathrm{msec}$. The stimuli were the same size as those in Experiments 2A and 2B, but they were presented on a PC using E-Prime.

\section{Results and Discussion}

The means across the blocks of the median correct RTs within each block are presented in Table 4, along with the percentages of errors. The RT data were analyzed in a two-factor repeated measures ANOVA with the factors being condition (box, line, or part-box) and consistency (consistent or inconsistent), with blocks as a random factor. There was a significant main effect of condition $[F(2,22)=15.35, p<.001]$, with word naming in the line condition tending to be overall faster than that in the other conditions. There was a main effect of consistency $[F(1,11)=20.30, p<.01]$ and also a condition $\times$ consis-

Table 4

Average Response Times (RTs, in Milliseconds) and Percentages of Correct Responses $(\% \mathrm{C})$ in the Three Context Conditions in Experiment 2C (Implicit Binding Task)

\begin{tabular}{lcccccccc}
\hline & \multicolumn{2}{c}{ Box } & & \multicolumn{2}{c}{ Line } & & \multicolumn{2}{c}{ Part-Box } \\
\cline { 2 - 3 } \cline { 8 - 9 } & RT & \%C & & RT & \%C & & RT & \%C \\
\hline Consistent & 1,064 & 86 & & 992 & 88 & & 1,092 & 85 \\
Inconsistent & 1,280 & 82 & & 1,006 & 88 & & 1,164 & 82 \\
\hline
\end{tabular}


tency interaction $[F(2,22)=5.55, p<.01]$. There was no effect of consistency when the word appeared with the line $[t(11)<1.0]$. There were reliable effects of consistency for both the box and the part-box conditions $[t(11)=$ -4.28 and -3.73 , both $p$ s $<.01$ ]. However, the size of the consistency effect was larger for the box than for the part-box condition; when these two conditions were entered along with consistency into a two-way ANOVA, there was a significant interaction $[F(1,11)=13.66, p<$ $.01]$. There were no reliable effects on errors and no signs of any speed-accuracy trade-offs.

These data replicate the results from Experiments 2A and $2 \mathrm{~B}$, while extending performance to include a further shape (the part-box). As before, G.K. showed an effect of the consistency of the position of the word within the box on naming times to the word, but no effect of spatial consistency when the word was presented along with the line. The results for the part-box condition fell between these two extremes; there was an effect of spatial consistency, but it was not as pronounced as when the word appeared in the box (the mean consistency effect was $216 \mathrm{msec}$ for the box stimulus and $72 \mathrm{msec}$ for the part-box).

These variations in the effects of spatial consistency may be attributed to the strength of binding in each of the conditions, with the word being bound more securely to the box than to the part-box and more tightly to the partbox than to the line, on the basis of the effects of common enclosure. Data on explicit binding in the three conditions, under unlimited exposure conditions, will be presented in Experiment 2D.

Finally, it should be noted that as in Experiment 1D, we established a contrast between implicit and explicit binding under brief durations (explicit binding at chance, along with reliable implicit binding), even though we used stimuli that were semantically related for both forms of binding (and in fact, the same stimuli were used for both forms in Experiment 2C). The difference between implicit and explicit binding cannot be attributed to a difference between binding related and unrelated elements.

\section{Experiment 2D \\ Explicit Binding of Words With Boxes, Lines, and Part-Boxes}

\section{Method}

The stimuli were the same as those in Experiment $2 \mathrm{C}$. There were 20 examples of each type of stimulus ( 2 words $\times 3$ contexts [box, line, or part-box] $\times 2$ spatial locations), creating 240 trials. G.K. was asked to read the word and to describe its spatial relation relative to the contextual stimulus (the box, part-box, or line stimuli). On each trial, a fixation cross was presented for $1 \mathrm{sec}$, followed by the display for an unlimited duration. Stimulus presentation here was controlled by E-Prime, and the stimuli appeared on a Pentium PC.

\section{Results and Discussion}

G.K. was able to make correct explicit decisions to $70 / 80(87.5 \%)$ of the words presented along with the box; in contrast, he made correct explicit decisions to only $52 / 80(65 \%)$ of the words with the lines and to $60 / 80$ $(75 \%)$ of the words in the part-boxes $\left[\chi^{2}(2)=11.10\right.$, $p<.01]$. Explicit spatial decisions were better when the words appeared within a box than when they appeared along with a separate horizontal line $\left[\chi^{2}(1)=11.18, p<\right.$ $.01]$ and relative to when they appeared within the partboxes $\left[\chi^{2}(1)=4.10, p<.05\right]$. The difference between the line and the part-box conditions was not reliable $\left[\chi^{2}(1)=\right.$ $1.91, p>.05]$. On all trials, G.K. reported that he was able to detect the presence of both stimuli.

Previous studies have demonstrated that G.K. is impaired at making explicit location decisions to stimuli (Humphreys \& Riddoch, 2003; Humphreys et al., 1994), and his performance when asked to decide on the location of the word relative to the line was consistent with this. His somewhat better performance when the word fell within the box likely reflects grouping of the word with the enclosing box, which helps to bind the spatial position of the word within the box (see also Shalev \& Humphreys, 2002, for other data on the effects of grouping on spatial judgments in G.K.). Presumably, there is still some, but weaker, binding between the word and the part-box, contrasting with little binding between the word and the line. More notably, the pattern of the data here follows that found in our tests of implicit spatial binding in Experiments $2 \mathrm{~A}-2 \mathrm{C}$; both explicit and implicit binding are affected in qualitatively similar ways by grouping relations between form stimuli.

\section{GENERAL DISCUSSION}

As in previous studies of patients with Balint's syndrome and impaired explicit binding of visual stimuli, we found that G.K. showed evidence of implicit binding (see also Robertson, 2003; Wojciulik \& Kanwisher, 1998). In the critical experiments here, we set G.K.'s ability to explicitly report the relations between the stimuli at chance level, using (relatively) short stimulus exposures. Nevertheless, his RTs for naming the colors of words were longer when the color was combined with an incongruent colored word (SOI condition) than when the color was combined with a congruent color word and separated from the incongruent color word (DOI condition; Experiments 1A, 1B, and 1D). Also, RTs for naming the word UP or DOwN were affected by the relative positions of the words within either a surrounding box or a part-box (RTs were shorter when the word was consistent with its relative spatial location than when it was inconsistent; Experiments $2 \mathrm{~A}$ and 2C). These results clearly indicate that patients can be sensitive to the spatial relations between colors and forms (Experiment 1) and between different forms (Experiment 2), even when their explicit judgments of these spatial relations are at chance.

In addition to this, we found that the size of Stroop interference in color naming varied with the spacing between the words presented in the experiment; interference was stronger when the words fell further apart than when they fell at close spatial locations (Experiment 1B vs. Experiment 1A; Experiment 1D). This effect of distance on implicit binding mirrored the effects of distance on explicit binding, when we compared explicit binding directly with close and spaced words (Experiment 1C). We also failed to find any evidence of implicit binding 
between a word and a horizontal line presented just above or below the word (Experiments 2B and 2C). This, too, mirrored the data on explicit binding. When G.K. had to report the location of a word relative to a surrounding box, a neighboring line, or a part-box, he was best when the word fell within the box (Experiment 2D). This was not a failure in detection, since he appeared able to discriminate the presence of both the word and the contextual stimulus (the box, line, or part-box) under the unlimited presentation conditions, but he was poor at coding relative position information, particularly for the word and the line. Apparently, both implicit and explicit binding of forms was affected by grouping between the elements; both implicit and explicit binding was greater when the word was grouped with the word by common enclosure.

On the basis of the similarities between implicit and explicit binding, we propose that these are not two qualitatively distinct processes. Instead, we propose that binding is a graded and not an all-or-none process, in which the strength of binding may decay over time and is a function of the attention focused on the stimuli. The role of attention may be to increase the strength of binding and, therefore, to prevent the binding from dissipating over time. In G.K., the strength of binding over time becomes very weak because of the unusually long period required for him to focus his attention on the stimuli. Therefore, when he finally detects the stimulus, binding information may be lost. However, for a short initial time, words and colors are correctly bound, on the basis of their co-location, and words and a surrounding box are bound by common enclosure. This initial binding is sufficient to generate RT differences for the naming of colors (in Experiment 1) and words (in Experiment 2).

We suggest that implicit and explicit binding alike are affected by such factors as the spacing between stimuli and the presence of Gestalt grouping cues. When two colored words were close together, G.K. tended to make increased numbers of ICs in explicit report tasks, in comparison with cases in which the words were spaced further apart (Experiment 1C). We suggest that ICs also arose when binding was tested implicitly (Experiments 1A, 1B, and 1D), when it had an impact on the magnitude of Stroop interference. In particular, ICs between the words would tend to decrease Stroop interference if ICs occur under conditions in which word components are not well coded. This would mean that the incongruent word identity is not then available to disrupt RTs to the critical color. In the tests of explicit binding between forms (Experiment 2D), we showed that binding decreased when there were minimal Gestalt cues for grouping a word and a neighboring line. This also arose in our study of implicit form binding (Experiments 2B and 2C). The data fit with the idea of a graded binding process, from initial binding by co-location and Gestalt grouping, to a subsequent more stable binding process contingent on spatial attention. In patients such as G.K., with impaired spatial attention, the information leading to initial, implicit binding can decay, leading to poor explicit binding performance. Importantly, both forms of binding are in- fluenced by similar visual processes, suggesting that they are not generated by distinct processing mechanisms.

\section{REFERENCES}

Cohen, A., \& Ivry, R. (1989). Illusory conjunctions inside and outside the focus of attention. Journal of Experimental Psychology: Human Perception \& Performance, 15, 650-663.

Cohen, A., \& Rafal, R. D. (1991). Attention and feature integration: Illusory conjunctions in a patient with a parietal lobe lesion. Psychological Science, 2, 106-110.

Cohen, J. D., Dunbar, K., \& McClelland, J. L. (1990). On the control of automatic processes: A parallel distributed-processing account of the Stroop effect. Psychological Review, 97, 332-361.

Cooper, A. C. G., \& Humphreys, G. W. (2000). Coding space within but not between objects: Evidence from Balint's syndrome. Neuropsychologia, 38, 723-733.

Friedman-Hill, S. R., Robertson, L. C., \& Treisman, A. (1995). Parietal contributions to visual feature binding: Evidence from a patient with bilateral lesions. Science, 269, 853-855.

Gilchrist, I. D., Humphreys, G. W., \& RidDOCH, M. J. (1996). Grouping and extinction: Evidence for low-level modulation of visual selection. Cognitive Neuropsychology, 13, 1223-1257.

Hall, D. A., Humphreys, G. W., \& Cooper, A. C. G. (2001). Neuropsychological evidence for case-specific reading: Multi-letter units in visual word recognition. Quarterly Journal of Experimental Psychology, 54A, 439-467.

Heywood, C. A., \& Cowey, A. (1999). Cerebral achromatopsia. In G. W. Humphreys (Ed.), Case studies in the neuropsychology of vision (pp. 17-40). Hove, U.K.: Psychology Press.

Heywood, C. A., \& ZIHL, J. (1999). Motion blindness. In G. W. Humphreys (Ed.), Case studies in the neuropsychology of vision (pp. 1-16). Hove, U.K.: Psychology Press.

Humphreys, G. W. (1998). Neural representations of objects in space: A dual coding account. Philosophical Transactions of the Royal Society of London: Series B, 353, 1341-1351.

Humphreys, G. W., Cinel, C., Wolfe, J., Olson, A., \& Klempen, N. (2000). Fractionating the binding process: Neuropsychological evidence distinguishing binding of form from binding of surface features. Vision Research, 40, 1569-1596.

Humphreys, G. W., \& RidDOCH, M. J. (2003). From what to where: Neuropsychological evidence for implicit interactions between object- and space-based attention. Psychological Science, 14, 487-492.

Humphreys, G. W., Riddoch, M. J., Nys, G., \& Heinke, D. (2002). Transient binding by time: Neuropsychological evidence from antiextinction. Cognitive Neuropsychology, 19, 361-380.

Humphreys, G. W., Romani, C., Olson, A., Riddoch, M. J., \& Duncan, J. (1994). Non-spatial extinction following lesions of the parietal lobe in humans. Nature, 372, 357-359.

Kornblum, S., \& Stevens, G. (2002). Sequential effects of dimensional overlap: Findings and issues. In W. Prinz \& B. Hommel (Eds.), Common mechanisms in perception and action (pp. 9-54). Oxford: Oxford University Press.

McClelland, J. L., \& Mozer, M. C. (1986). Perceptual interactions in two-word displays: Familiarity and similarity effects. Journal of Experimental Psychology: Human Perception \& Performance, 12, 18-35.

Moran, J., \& Desimone, R. (1985). Selective attention gates visual processing in the extrastriate cortex. Science, 229, 782-784.

PAlmer, S., \& Rock, I. (1994). Rethinking perceptual organization: The role of uniform connectedness. Psychonomic Bulletin \& Review, 1, 29-55.

Robertson, L. C. (2003). Binding, spatial attention and perceptual awareness. Nature Reviews Neuroscience, 4, 93-102.

Robertson, L. C., Treisman, A., Friedman-Hill, S., \& GraboWECKY, M. (1997). The interaction of spatial and object pathways: Evidence from Balint's syndrome. Journal of Cognitive Neuroscience, $\mathbf{9}$, 295-317.

Shalev, L., \& Humphreys, G. W. (2002). Implicit location encoding via stored representations of familiar objects: Neuropsychological evidence. Cognitive Neuropsychology, 19, 721-744. 
SingER, W., \& GRAY, C. M. (1995). Visual feature integration and the temporal correlation hypothesis. Annual Review of Neuroscience, 18, 555-586.

Treisman, A. (1998). Feature binding, attention and object perception. Philosophical Transactions of the Royal Society of London: Series B, 353, 1295-1306.

Treisman, A., \& Schmidt, H. (1982). Illusory conjunction in the perception of objects. Cognitive Psychology, 14, 107-141.
WARD, R., DAnziger, S., Owen, V., \& Rafal, R. D. (2002). Deficits in spatial coding and feature binding following damage to spatiotopic maps in the human pulvinar. Nature Neuroscience, 5, 99-100.

WoJCIUlik, E., \& KANWISHER, N. (1998). Implicit but not explicit feature binding in a Balint's patient. Visual Cognition, 5, 157-181.

(Manuscript received April 25, 2003;

revision accepted for publication November 14, 2004.) 